\title{
Quantum efficiency enhancement optimization in colloidal semiconductor quantum dot solids using nonradiative energy transfer
}

\author{
Sedat Nizamoglu, Onur Akin and Hilmi Volkan Demir \\ Department of Electrical and Electronics Engineering, Department of Physics, Nanotechnology Research Center, and Institute of Materials \\ Science and Nanotechnology, Bilkent University, Ankara, Turkey TR-06800 \\ Email: volkan@bilkent.edu.tr
}

\begin{abstract}
We investigate quantum efficiency (QE) enhancement via recycling of trapped excitons in energy-gradient of quantum dot solids using nonradiative energy transfer. The maximum QE increase of $17 \%$ is achieved when the donor-acceptor ratio is $1: 1$.
\end{abstract}

(C)2010 Optical Society of America

OCIS codes: (160.4236) Nanomaterials; (160.4760) Optical properties

Colloidal semiconductor quantum dots (QDs) possess favorable characteristics to serve as color-converters in hybrid white light emitting diodes (LEDs). For instance, red-emitting QDs accomplish a photon conversion efficiency of $52.8 \%$, which is higher than that of their phosphor-based counterparts [1]. These QDs exhibit strong absorption of incident blue/UV pump light and in turn make photon emission for color conversion at the required photon energy that is precisely tuneable. Furthermore, such QDs cover the entire emission spectrum from visible to infrared using the size effect. Their high-quality solid films can easily be formed using standard techniques including layer-by-layer film deposition and spin casting [2]. Because of these advantages, QD integrated LEDs achieved important steps forward in solid state lighting such as highly color-rendered, warm lighting. However, these QDs experience a reduction in quantum efficiency (QE) in solid film with respect to their higher QE in solution [3]. To address this problem, trapped excitons can be recycled into QDs via nonradiative energy transfer (ET) (i.e., Förster-type energy transfer), which offers a potential increase in the QE of these QD solid films. Here we present the optimization of the donor-acceptor (D-A) QD ratios to investigate QE enhancement in these colloidal QD solids [4]. To have nonradiative ET, an energy gradient QD system is needed. For that blends of cyan- and green-emitting CdSe/ZnS core/shell QDs $\left(\lambda_{\mathrm{PL}}=500 \mathrm{~nm}\right.$ and $550 \mathrm{~nm}$, respectively) are used as the respective D and A ET pairs. D-A ratios of 16:1, 4:1, 1:1, 1:4 and 1:16 are investigated. All of these D-A ratios are experimentally demonstrated to show QE enhancement by comparing against their control groups of donors only and acceptors only (Figure 1).

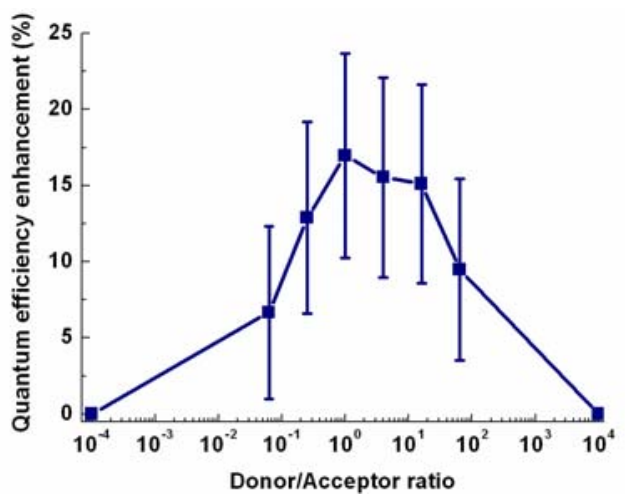

Figure 1. Quantum efficiency enhancement as a function of the donor-acceptor QD ratio.

For quantum efficiency measurements, a monochromator (Newport) with a broad-emitting light source is used to pump QDs and an integrating sphere (Newport) that collects the entire luminescence of these QDs is connected to the monochromator. For time-resolved luminescence decay measurements, a spectroscopy system (Picoquant) is used with a calibrated time-resolution of 16 ps. Figure 1 presents the QE enhancement measurements of these QDs dependent on their D-A ratios compared to the control groups (which are represented as the base line at the far edges of the plot for easier visualization). In all of these energy gradient QD structures with different donor-acceptor 


\section{CTuNN6.pdf}

ratios, a QE enhancement is experimentally observed. To analyze these results, Equation 1 shows the expected overall quantum efficiency of the hybrid film $\left(\mathrm{QE}_{\text {hybrid }}\right)$, which is a weighted sum of the $\mathrm{QE}$ of acceptors $\left(\mathrm{QE}_{\mathrm{A}}\right)$ and that of the donors $\left(\mathrm{QE}_{\mathrm{D}}\right)$. According to Equation (1), $\mathrm{QE}_{\text {hybrid }}$ is expected to lie between $\mathrm{QE}_{\mathrm{A}}$ and $\mathrm{QE}_{\mathrm{D}}$, and it is therefore not possible to increase the overall $\mathrm{QE}$ of the hybrid film higher than that of the donors or that of the acceptors. However, the experiments reproducibly demonstrate the opposite that $\mathrm{QE}_{\text {hybrid }}$ in fact exceeds both $\mathrm{QE}_{\mathrm{A}}$ and $\mathrm{QE}_{\mathrm{D}}$. This experimental observation is attributed to the recycling of trapped excitons. Following the optical absorption of the incident pump, the photogenerated excitons are expected to relax to their ground states; but, due to the existing surface defects, some of the excitons are trapped into these defect states and subsequently typically make nonradiative recombination. Via ET, however, these excitons trapped in the defect states can also be transferred, contributing to the quantum efficiency enhancement. Thus, by recycling the excitons in trap states, the emission of the QD D-A solids with energy gradient can be increased beyond the quantum efficiency of the donors or acceptors alone.

$$
\mathrm{QE}_{\mathrm{hybrid}}=\mathrm{QE}_{\mathrm{A}} \times\left(1+\tau_{\mathrm{ET}} / \tau_{\mathrm{D}}\right)^{-1}+\mathrm{Q}_{\mathrm{ED}} \times\left(1+\tau_{\mathrm{D}} / \tau_{\mathrm{ET}}\right)^{-1}
$$

Figure 2 shows the time-resolved photoluminescence of the hybrid QD D-A ET sample with a D-A ratio of 1:1 (exhibiting the peak luminescence enhancement) along with that of the only cyan-emitting donor nanocrystal solids as the control group, both at $500 \mathrm{~nm}$. The emission of the ET sample decays faster in comparison with the only cyan-emitting donors at $500 \mathrm{~nm}$ because these cyan-emitting QDs transfer their excitation energy to green-emitting QDs in the hybrid film. Also, as shown in the inset of Figure 2, at $550 \mathrm{~nm}$, the ET sample decays slower because of taking energy from the donors. We analyze these emission decays in Figure 2 using a multiexponential model fit. According to our numerical analysis, the lifetime calculated from the donor decay is $4.4 \mathrm{~ns}$ and the efficiency of the nonradiative ET exhibits a relatively high level of $48 \%$. But, the lifetime calculated for the acceptors is $14.1 \mathrm{~ns}$, which is higher than the lifetime of the donors, because of the additional ET of the trapped excitons into the acceptor QDs.

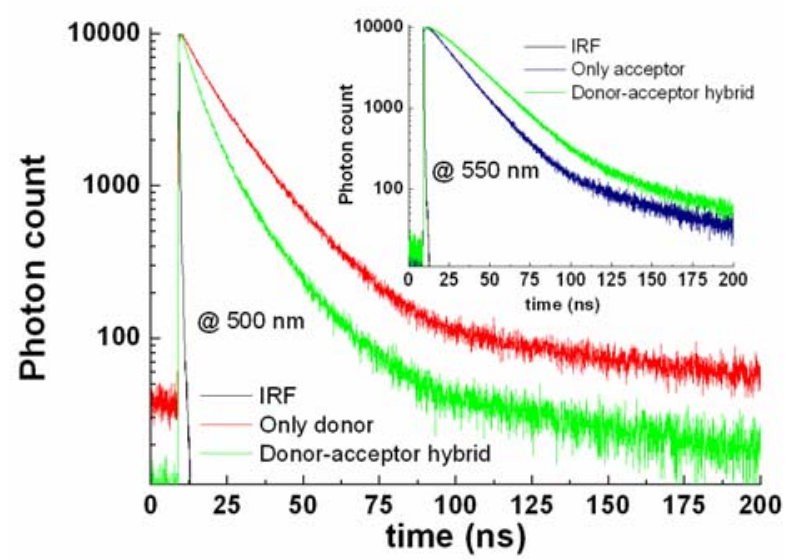

Figure 2. Time-resolved photoluminescence decay curves of the ET sample with a D-A ratio 1:1 and only donor QDs at $500 \mathrm{~nm}$, along with those of the same ET samples and only donor QDs at $550 \mathrm{~nm}$ shown in the inset.

In conclusion, blended QDs of various D-A ratios in solid film have been investigated and the D-A ratio of 1:1 has been found to exhibit the peak QE enhancement of 17\% by recycling of their trapped excitons in the solid film.

This work is supported by EU PHOREMOST Network of Excellence and EU Nanophotonics4Energy Network of Excellence, TUBITAK EEEAG No. 109E002, 109E004, 106E020, 104E114, 107E088, and 107E297. HVD also acknowledges ESF-EURYI and TUBA-GEBIP.

\section{References}

[1] D. Ming. Yeh, C. F. Huang, Y. C. Lu, and C. C. Yang, "White-light light-emitting device based on surface plasmon-enhanced CdSe/ZnS nanocrystal wavelength conversion on a blue/green two-color light-emitting diode,” Appl. Phys. Lett. 92, 091112 (2008).

[2] M. Achermann, M. A. Petruska, S. Kos, D. L. Smith, D. D. Koleske and V. I. Klimov, “ Energy- transfer pumping of semiconductor nanocrystals using an epitaxial quantum well," Nature 429, 642 (2004).

[3] A. A. Chistyakov, I. L. Martynov, K. E. Mochalov, V. A. Oleinikov, S. V. Sizova, E. A. Ustinovich, and K. V. Zakharchenko, "Interaction of CdSe/ZnS core-shell semiconductor nanocrystals in solid thin films," Laser Phys. 16, 1625 (2006).

[4] S. Nizamoglu, O. Akin, and H. V. Demir, "Quantum efficiency enhancement in nanocrystal solids using nanoradiative energy transfer with optimized donor-acceptor ratio for hybrid white light emitting diodes," Appl. Phys. Lett. 94, 243107 (2009). 